\title{
Large venous malformation of right colonic flexure
}

\author{
Akihiro Kitahama ${ }^{1}$, Yasumi Katayama ${ }^{1}$, Yoshitake Sugamata ${ }^{2}$, and Masaya Tamano ${ }^{1}$
}

Departments of ${ }^{1}$ Gastroenterology and ${ }^{2}$ Surgery, Dokkyo Medical University Koshigaya Hospital, Koshigaya, Japan
Received: March 12, 2016

Revised : April 14, 2016

Accepted: April 14, 2016

\section{Correspondence to}

Yasumi Katayama, M.D.

Tel: +81-48-965-1111

Fax: +81-48-965-1169

E-mail:yasumi@dokkyomed.ac.jp
Colonoscopy of a 38-year-old woman with persistent diarrhea despite drug therapy revealed no inflammatory changes in the colonic mucosa, but a dark blue, elevated lesion with a diameter of $3 \mathrm{~cm}$ was incidentally discovered in the right colonic flexure (Fig. $1 \mathrm{~A}$ ). Computed tomography showed vascular lesions with a clear boundary, 3 $\mathrm{cm}$ in diameter, with a patchy contrast effect in the right colonic flexure. The late arterial phase of angiography revealed a scattered light cotton-like stain that was consistent with the location of the lesion and vein flow into the lesion was noticeable during the venous phase (Fig. 1B). According to the International Society for the Study of Vascular Anomalies (ISSVA) classification, this lesion was diagnosed as a venous malformation (formerly known as a cavernous hemangioma). Intervention therapy is not considered suitable for this type of vascular anomaly, but therapy such as endoscopic mucosal resection has been described for venous malformations. However, the size of the lesion and the shape of the base precluded endoscopic therapy. The patient was therefore treated by laparoscopic ascending colon resection. Histologically, vascular endothelial cells had proliferated from the submucosa through the muscle layer and subserosa, which was consistent with a venous malformation (Fig. 2).

\section{Conflict of interest}

No potential conflict of interest relevant to this article was reported.
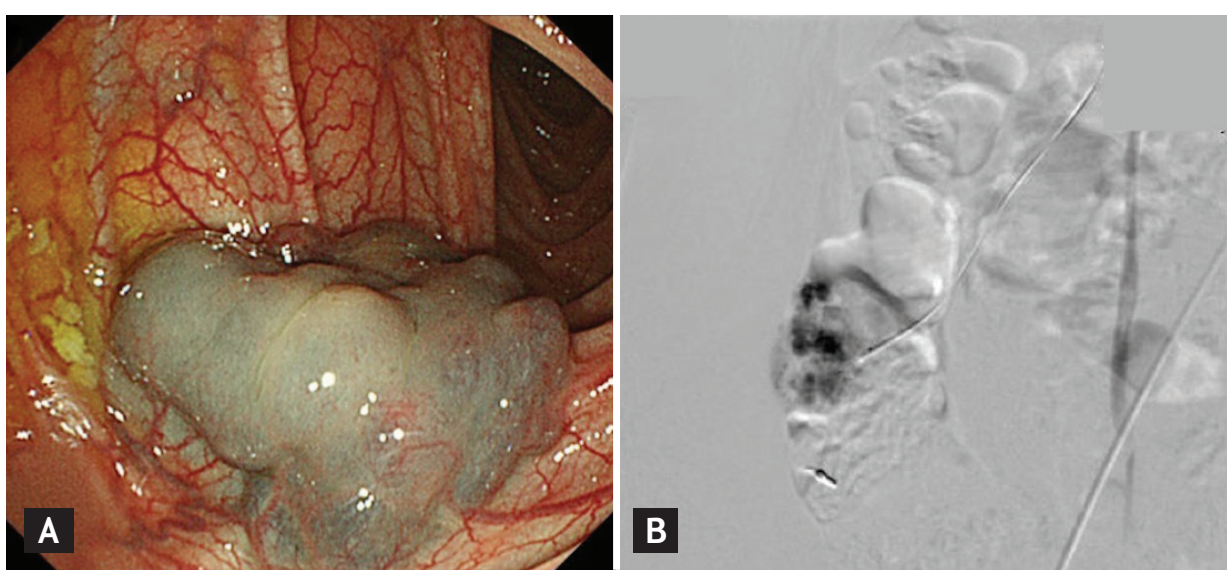

Figure 1. (A) Colonoscopy showing a dark blue elevated lesion in the right colonic flexure. (B) Abdominal angiography showing scattered light cotton-like stain of the lesion in the late arterial phase. 


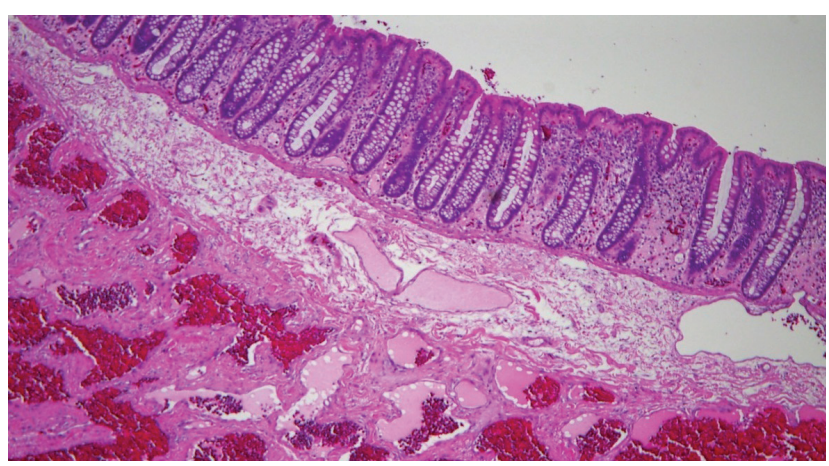

Figure 2. Microphotograph showing vascular endothelial cells proliferated from the submucosa through the muscle layer and subserosa $(\mathrm{H} \& \mathrm{E}, \times 100)$. 